\title{
The dog as an animal model for DISH?
}

\author{
H. C. Kranenburg • L. A. Westerveld · J. J. Verlaan • \\ F. C. Oner • W. J. A. Dhert • G. Voorhout • \\ H. A. W. Hazewinkel · B. P. Meij
}

Received: 23 September 2009/Revised: 2 December 2009/Accepted: 12 January 2010/Published online: 2 February 2010

(C) The Author(s) 2010. This article is published with open access at Springerlink.com

\begin{abstract}
Diffuse idiopathic skeletal hyperostosis (DISH) is a systemic disorder of the axial and peripheral skeleton in humans and has incidentally been described in dogs. The aims of this retrospective radiographic cohort study were to determine the prevalence of DISH in an outpatient population of skeletally mature dogs and to investigate if dogs can be used as an animal model for DISH. The overall prevalence of canine DISH was 3.8\% (78/2041). The prevalence of DISH increased with age and was more frequent in male dogs, similar to findings in human studies. In the Boxer breed the prevalence of DISH was $40.6 \%$ (28/69). Dog breeds represent closed gene pools with a high degree of familiar relationship and the high prevalence in the Boxer may be indicative of a genetic origin of DISH. It is concluded that the Boxer breed may serve as an animal model for DISH in humans.
\end{abstract}

Keywords DISH $\cdot$ Spine $\cdot$ Dog $\cdot$ Prevalence $\cdot$ Boxer

\section{Introduction}

Diffuse idiopathic skeletal hyperostosis (DISH) is a systemic disease of the axial and peripheral skeleton [10]. DISH results in ossification of soft tissues including the

H. C. Kranenburg · W. J. A. Dhert · G. Voorhout .

H. A. W. Hazewinkel · B. P. Meij ( $\square)$

Department of Clinical Sciences of Companion Animals, Faculty of Veterinary Medicine, Utrecht University, Yalelaan 108, PO Box 80154, 3508 TD Utrecht, The Netherlands e-mail: B.P.Meij@uu.nl

L. A. Westerveld · J. J. Verlaan · F. C. Oner - W. J. A. Dhert Department of Orthopaedics, University Medical Center Utrecht, Utrecht, The Netherlands longitudinal spinal ligaments and sites of attachment of tendons and capsules to bone. DISH was first described by Forestier and Rotes-Querol in 1950 [10]. In 1976, Resnick and Niwayama [28] postulated three criteria for the diagnosis of DISH which are now widely used in literature and may be helpful in differentiating DISH from other spinal disorders, such as spondylosis deformans, intervertebral osteochondrosis and ankylosing spondylitis. The prevalence of DISH varies with geographic location and ranges from $2.9 \%$ in Korea to $25 \%$ in the USA [13, 14, 32, 34, 35]. The variation in prevalence for DISH found throughout the world suggests a possible genetic and/or ethnic factor in the pathogenesis of DISH [6, 13, 34]. The etiologic factors are not yet clear but various metabolic, endocrinological, genetic and environmental factors have been suggested to contribute to its development [15, 18, 21]. DISH is associated with obesity and the incidence increases with body weight in both the genders $[18,20,21]$. DISH often remains asymptomatic but can lead to mild back pain, stiffness and restriction in spinal range of motion [3, 23]. In advanced stages of DISH, multiple level spinal ankylosis may lead to spinal cord compression or result in unstable fractures of the spine, even after trivial trauma, often mimicking clinical features of ankylosing spondylitis [4, 32].

DISH has been described in two canine cases, in which both dogs showed orthopedic and neurological abnormalities and were eventually euthanized because of the severity of symptoms [22, 37]. Morgan and Stavenborn suggested that DISH and extensive forms of spondylosis deformans in dogs, a condition frequently described in veterinary literature, have many features in common $[5,17,22]$.

For more than 15,000 years dogs have lived with humans in comparable conditions [29,33]. They share the 
same environment and are subjected to similar nutritional and toxic substances. The nutrition of dogs on commercial pet food diets has been researched intensively [8]. But yet a growing number of dogs develop obesity and estimations vary between 20 and $40 \%$ [11]. Endocrinological syndromes and conditions of the spine show great similarities between humans and dogs, and for specific diseases in humans, dogs may serve as a spontaneous disease animal model [2, 9, 16, 19]. However, no animal model has been described for DISH. Therefore the aims of the present study were: (1) to determine the prevalence and spinal distribution of DISH in an outpatient population of skeletally mature dogs, (2) to study the relation between DISH and age, gender, neutering status and breed and (3) to investigate if dogs could be used as an animal model for DISH.

\section{Materials and methods}

Radiographs of dogs older than 1 year, referred to the Department of Clinical Sciences of Companion Animals between February 2003 and January 2008, were retrieved from the hospital database. From this cohort, radiographs were selected when more than 20 dogs of a particular breed were available, leading to a total of $2041 \mathrm{dogs}$ from 33 breeds. All dogs were referred by veterinarians for various medical conditions not necessarily related to the spine.

Radiographs of the thorax, abdomen and cervical and/ or thoracolumbar spine were all eligible for review. DISH was confirmed when the criteria of Resnick and Niwayama were met [28]. The radiographs were screened by the principal investigator according to a predefined scoring system: (1) DISH, (2) possible DISH and (3) no DISH. Radiographs marked with score 1 or 2 were re-reviewed by two investigators in a consensus meeting to confirm or refute the diagnosis of DISH. When consensus on the diagnosis was not established, a third investigator was consulted. The indications for radiographic examination were reviewed and categorized in three groups. Suspected thoracic abnormalities and screening for metastatic disease constituted the majority of indications $(75 \%)$. The group 'spinal' included dogs that were examined for neurological or orthopaedic complaints $(18 \%)$. In $7 \%$ the indication for the radiographs was an abdominal problem.

Statistical analysis was performed using SPSS software (version 16.0). The Student's $t$-test was performed on the mean ages of dogs with and without DISH. Univariate analysis was performed on age, gender, neutering status, breed in general and in case one or more breed(s) appeared most affected, for a particular breed separately. Variables that were significantly related to DISH in the univariate analysis were subsequently analyzed in a multiple logistic regression model. Significance was set if $p<0.05$.

\section{Results}

From the total of $2041 \mathrm{dogs}, 51.3 \%$ were male and $48.7 \%$ female. The mean age $\pm \mathrm{SE}$ of all dogs was $7.2 \pm$ 0.2 years (range 1-17 years). The mean age of dogs with DISH (8.9 \pm 1.0 years, range $2-16$ years) was significantly ( $p=0.00$ ) higher than the mean age of dogs without DISH (7.0 \pm 0.2 ; range $1-17$ years). The overall prevalence of DISH in this cohort was $3.8 \% \quad(78 / 2041)$ (95\% confidence interval (CI) 3.0-4.7) (Table 1). DISH was present in male dogs more frequently than in female dogs. In male dogs the prevalence of DISH was $4.3 \%$, in female dogs the prevalence was $3.3 \%$. Stratified into three age groups, DISH was more prevalent in older dogs (Table 1).

Fifteen breeds were affected by DISH. Among these, only the Boxer breed showed an exceptionally high prevalence and reliable confidence interval: $40.6 \%(28 / 69)$ (95\% CI 29.0-52.2). In contrast, two other popular dog breeds, the Labrador Retriever and the Bernese Mountain dog, that were well represented in the cohort, showed a low prevalence for DISH (2.5 and $2.0 \%$, respectively) (Table 2). In dogs of 18 other breeds, including the Beagle
Table 1 The prevalence of DISH in 2041 dogs according to gender and age groups: number of dogs (percentage; 95\% confidence interval)

\begin{tabular}{lrlr}
\hline & \multicolumn{1}{l}{ No DISH } & \multicolumn{1}{l}{ DISH } & \multicolumn{1}{c}{ Total } \\
\hline Gender & & & \\
$\quad$ Male dogs & $1,002(95.7 ; 94.5-96.9)$ & $45(4.3 ; 3.1-5.5)$ & $1,047(100.0)$ \\
Female dogs & $961(96.7 ; 95.6-97.8)$ & $33(3.3 ; 2.2-4.4)$ & $994(100.0)$ \\
Age group (years) & & & $749(100.0)$ \\
$1-5$ & $736(98.3 ; 97.3-99.2)$ & $13(1.7 ; 0.8-2.7)$ & $993(100.0)$ \\
$6-10$ & $951(95.8 ; 94.5-97.0)$ & $42(4.2 ; 3.0-5.5)$ & $299(100.0)$ \\
$11-17$ & $276(92.3 ; 89.3-95.3)$ & $23(7.7 ; 4.7-10.7)$ & $2,041(100.0)$ \\
Total & $1,963(96.2 ; 95.4-97.0)$ & $78(3.8 ; 3.0-4.7)$ &
\end{tabular}


Table 2 Prevalence of DISH (number ( $\% ; 95 \%$ confidence interval) in eight large dog breeds $(>25 \mathrm{~kg})$ of which at least 60 dogs were available for screening

\begin{tabular}{lcc}
\hline Breed & $N$ & DISH \\
\hline Labrador Retriever & 244 & $6(2.5 ; 0.5-4.4)$ \\
Bernese Mountain dog & 199 & $4(2.0 ; 0.1-4.0)$ \\
Golden Retriever & 156 & $6(3.9 ; 0.8-6.9)$ \\
Rottweiler & 120 & $2(1.7 ;-0.6-4.0)$ \\
German Shepherd & 113 & $8(7.1 ; 2.4-11.8)$ \\
Boxer & 69 & $28(40.6 ; 29.0-52.2)$ \\
Bouvier des Flandres & 63 & $4(6.4 ; 0.3-12.4)$ \\
Flatcoated Retriever & 61 & $8(13.1 ; 4.6-21.6)$ \\
\hline
\end{tabular}

(a dog frequently used in experimental studies), DISH was not found.

DISH was predominantly localized in the thoracolumbar spine (Fig. 1). Two dogs showed DISH in the cervical spine, 52 dogs in the thoracic spine, 22 dogs in the thoracolumbar spine (Fig. 2) and two dogs in the lumbar spine (Fig. 3).

Univariate analysis on parameters age, gender, neutering status and breed resulted in a statistically significant positive odds ratio of 1.22 for age only (95\% CI $1.14-1.31$, $p=0.00)$. The odds ratio's for gender of $1.31(95 \% \mathrm{CI}$ $0.83-2.07, p=0.25)$ and neutering status of $1.09(95 \% \mathrm{CI}$ $0.69-1.71, p=0.72)$ were not significant $(p>0.05)$. The odds ratio for the most affected dog breeds was investigated and univariate analysis resulted in a significant positive odds ratio for the dog breed Boxer of 26.25 (95\% CI $15.05-45.80, p=0.00$ ).

The parameters significantly related to DISH in the univariate analysis were subsequently analyzed in a multiple logistic regression model. This model showed that age (1.32, 95\% CI 1.21-1.44) and dog breed Boxer (51.27, 95\% CI 26.57-98.93) were significantly related to the presence of DISH ( $p=0.00$ for all parameters).

\section{Discussion}

Similar to humans, male dogs were more frequently affected by DISH than female dogs and the overall prevalence increased with age. A genetic background of DISH in humans was suggested to underlie the difference of the prevalence in ethnic groups with Caucasians being most frequently affected, compared to native Africans, AfroAmericans and Asians [6, 7, 13, 34]. The Boxer breed, like many inbred dog breeds, represents a closed gene pool with a high degree of familiar relationships $[25,26]$. The high prevalence of a specific disease in a certain dog breed and absence of this disease in other breeds, is suggestive of a genetic mechanism [24]. The high prevalence of DISH in the Boxer breed may allow for the study of DISH in a spontaneous disease animal model. With the present knowledge of the sequenced canine (Boxer) genome $[19,25,26]$, future studies could focus on the elucidation of the gene or genes involved in the pathogenesis of DISH
Fig. 1 Distribution of bridging ossifications of the spinal with DISH longitudinal ligament in $78 \mathrm{dogs}$

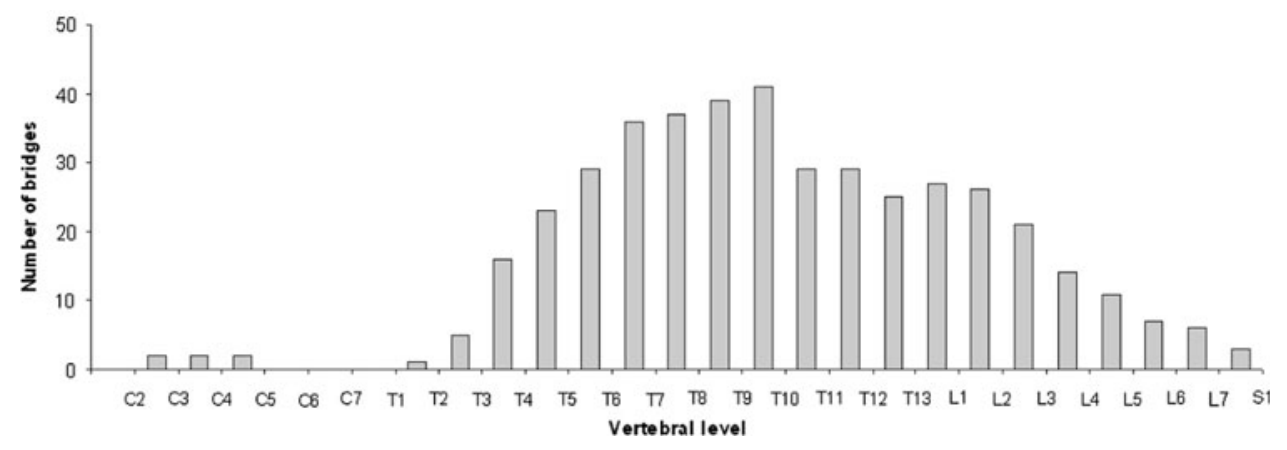

Fig. 2 Radiograph of an 8-year-old neutered female Boxer with DISH at T9-L7

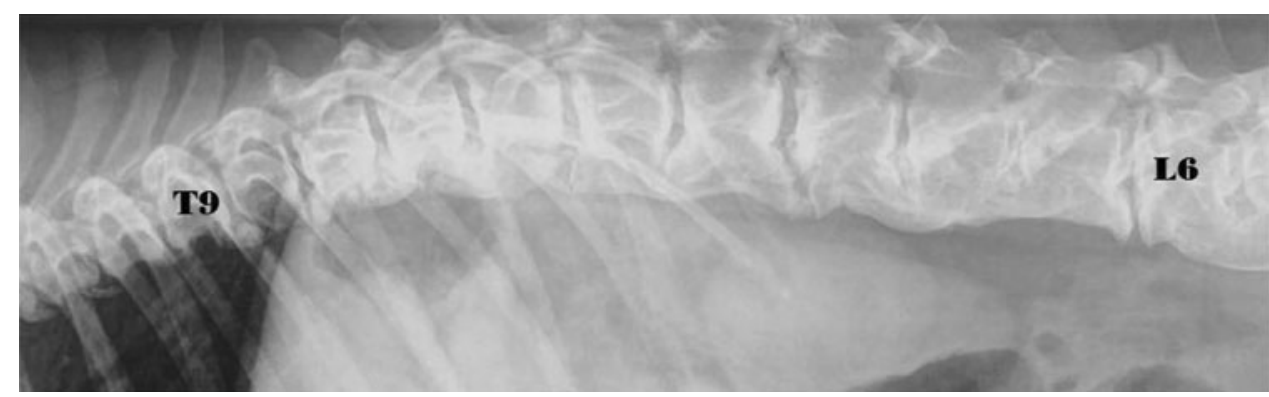


Fig. 3 Radiograph of an 8-year-old neutered male Boxer with DISH at L4-S1

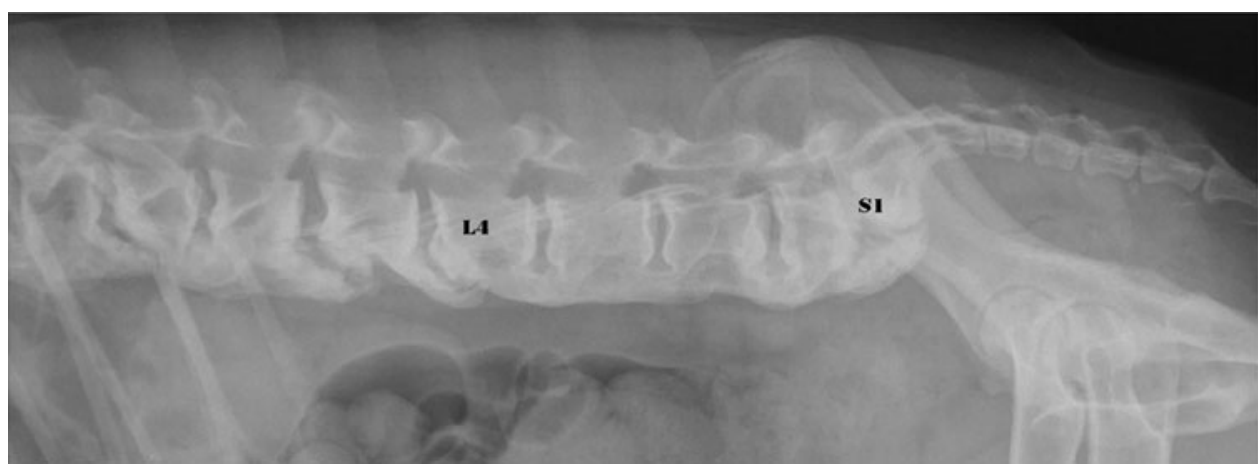

using association studies with single nucleotide polymorphisms at high density [12].

When using the $\operatorname{dog}$ as an animal model for spinal research some biomechanical issues are important to consider. For example the direction of the gravity load on the spine in quadruped dogs is not the same as in bipedal humans and therefore the loading on the human spine is commonly assumed to be larger than that in quadrupeds. However, biomechanical measurements show the contrary. The muscle contraction forces and tension structures such as ligaments and intervertebral discs in the quadruped spine add substantially to the axial loading on the spine [36] which is actually larger in quadrupeds than in bipeds $[1,31]$. This may limit how we extrapolate the results of canine experiments to humans [1]. The canine spine is loaded along its long axis just like the human spine [31]. The dog may serve as a valuable animal model for spinal research but, as with all animal models, care must be taken when transferring the results to the human situation $[1,30,31]$.

The segmental distribution of DISH in the spine in our cohort of dogs showed great similarities to that in humans. Resnick and Niwayama reported in their study of 215 cadaveric spines and 100 patients with spinal manifestations of DISH that the 7th to 10th thoracic vertebrae were most frequently affected, although lumbar involvement was also present. In addition, they noted a lower incidence in the most cranial thoracic vertebrae [2]. In the present study in dogs, DISH was found in similar locations as in humans. The caudal thoracic vertebrae and, to a lesser degree, the cranial lumbar vertebrae were most frequently affected in dogs.

No studies on DISH in dogs have been reported other than the case reports of Morgan and Stavenborn and Woodard et al. Both dogs were eventually euthanized because of extreme stiffness and pain of the spine and joints refractory to medical treatment $[22,37]$. The bone formation along the spine in DISH probably originates from ossification/calcification of the anterior (or, in quadrupeds, ventral) longitudinal ligament whereas spondylosis deformans originates from the endplates of vertebral bodies. Spondylosis deformans is a common disorder in dogs and prevalences of spondylosis deformans ranging from 26 to $50 \%$ in Boxers have been published $[5,17]$. The radiographic differentiation of both conditions, however, remains difficult in severe cases [27]. Morgan and Stavenborn suggested that DISH in dogs closely resembled extensive spondylosis deformans. In retrospect, it may have been more appropriate to use the term DISH for some of the severe cases of spondylosis deformans reported in dogs [22].

In conclusion, the current radiographic study showed the presence of DISH in some dog breeds and demonstrated that its prevalence increased with age and was higher in male dogs. Considering the unusually high prevalence of DISH in the Boxer breed, this animal may serve as a spontaneous disease animal model for DISH in humans. Furthermore, our results are indicative of a genetic origin of canine DISH. Future research may be directed at the elucidation of the gene(s) involved in the pathogenesis of this disorder.

Open Access This article is distributed under the terms of the Creative Commons Attribution Noncommercial License which permits any noncommercial use, distribution, and reproduction in any medium, provided the original author(s) and source are credited.

\section{References}

1. Alini M, Eisenstein SM, Ito K et al (2008) Are animal models useful for studying human disc disorders/degeneration? Eur Spine J 17:2-19

2. An HS, Masuda K (2006) Relevance of in vitro and in vivo models for intervertebral disc degeneration. J Bone Joint Surg Am 88(Suppl 2):88-94

3. Belanger TA, Rowe DE (2001) Diffuse idiopathic skeletal hyperostosis: musculoskeletal manifestations. J Am Acad Orthop Surg 9:258-267

4. Callahan EP, Aguillera H (1993) Complications following minor trauma in a patient with diffuse idiopathic skeletal hyperostosis. Ann Emerg Med 22:1067-1070

5. Carnier P, Gallo L, Sturaro E et al (2004) Prevalence of spondylosis deformans and estimates of genetic parameters for the degree of osteophytes development in Italian Boxer dogs. J Anim Sci 82:85-92

6. Cassim B, Mody GM, Rubin DL (1990) The prevalence of diffuse idiopathic skeletal hyperostosis in African blacks. Br J Rheumatol 29:131-132 
7. Childs SG (2004) Diffuse idiopathic skeletal hyperostosis: Forestier's disease. Orthop Nurs 23:375-382

8. National Research Council (2006) Ad Hoc Committee on Dog and Cat Nutrition. Nutrient requirements of dogs and cats. The National Academies Press, Washington, DC

9. de Bruin C, Meij BP, Kooistra HS et al (2009) Cushing's disease in dogs and humans. Horm Res 71(Suppl 1):140-143

10. Forestier J, Rotes-Querol J (1950) Senile ankylosing hyperostosis of the spine. Ann Rheum Dis 9:321-330

11. German AJ (2008) The growing problem of obesity in dogs and cats. J Nutr 136:1940S-1946S

12. Karlsson EK, Lindblad-Toh K (2008) Leader of the pack: gene mapping in dogs and other model organisms. Nat Rev Genet 9:713-725

13. Kim SK, Choi BR, Kim CG et al (2004) The prevalence of diffuse idiopathic skeletal hyperostosis in Korea. J Rheumatol 31:2032-2035

14. Kiss C, O'Neill TW, Mituszova M et al (2002) The prevalence of diffuse idiopathic skeletal hyperostosis in a population-based study in Hungary. Scand J Rheumatol 31:226-229

15. Kiss C, Szilagyi M, Paksy A et al (2002) Risk factors for diffuse idiopathic skeletal hyperostosis: a case-control study. Rheumatology (Oxford) 41:27-30

16. Kooistra HS, Galac S, Buijtels JJ et al (2009) Endocrine diseases in animals. Horm Res 71(Suppl 1):144-147

17. Langeland M, Lingaas F (1995) Spondylosis deformans in the boxer: estimates of heritability. J Small Anim Pract 36:166-169

18. Li H, Jiang LS, Dai LY (2007) Hormones and growth factors in the pathogenesis of spinal ligament ossification. Eur Spine $\mathrm{J}$ 16:1075-1084

19. Lindblad-Toh K, Wade CM, Mikkelsen TS et al (2005) Genome sequence, comparative analysis and haplotype structure of the domestic dog. Nature 438:803-819

20. Mader R, Dubenski N, Lavi I (2005) Morbidity and mortality of hospitalized patients with diffuse idiopathic skeletal hyperostosis. Rheumatol Int 26:132-136

21. Mata S, Fortin PR, Fitzcharles MA et al (1997) A controlled study of diffuse idiopathic skeletal hyperostosis. Clinical features and functional status. Medicine (Baltimore) 76:104-117

22. Morgan JP, Stavenborn M (1991) Disseminated idiopathic skeletal hyperostosis (DISH) in a dog. Vet Radiol Ultrasound 32:65-70
23. Olivieri I, D’Angelo S, Cutro MS et al (2007) Diffuse idiopathic skeletal hyperostosis may give the typical postural abnormalities of advanced ankylosing spondylitis. Rheumatology (Oxford) 46:1709-1711

24. Ostrander EA, Galibert F, Patterson DF (2000) Canine genetics comes of age. Trends Genet 16:117-124

25. Ostrander EA, Wayne RK (2005) The canine genome. Genome Res 15:1706-1716

26. Parker HG, Ostrander EA (2005) Canine genomics and genetics: running with the pack. PLoS Genet 1:e58

27. Resnick D (1985) Degenerative diseases of the vertebral column. Radiology 156:3-14

28. Resnick D, Niwayama G (1976) Radiographic and pathologic features of spinal involvement in diffuse idiopathic skeletal hyperostosis (DISH). Radiology 119:559-568

29. Savolainen P, Zhang Y, Luo J et al (2002) Genetic evidence for an East Asian origin of domestic dogs. Science 298:1610-1613

30. Schimandle JH, Boden SD (1994) Spine update. Animal use in spinal research. Spine 19:2474-2477

31. Smit TH (2002) The use of a quadruped as an in vivo model for the study of the spine-biomechanical considerations. Eur Spine J 11:137-144

32. Sreedharan S, Li YH (2005) Diffuse idiopathic skeletal hyperostosis with cervical spinal cord injury-a report of 3 cases and a literature review. Ann Acad Med Singapore 34:257-261

33. Vilà C, Savolainen P, Maldonado J et al (1997) Multiple and ancient origins of the domestic dog. Science 276:1687-1689

34. Weinfeld RM, Olson PN, Maki DD et al (1997) The prevalence of diffuse idiopathic skeletal hyperostosis (DISH) in two large American Midwest metropolitan hospital populations. Skeletal Radiol 26:222-225

35. Westerveld LA, van Ufford HM, Verlaan JJ et al (2008) The prevalence of diffuse idiopathic skeletal hyperostosis in an outpatient population in The Netherlands. J Rheumatol 35:16351638

36. Wilke H, Rohlmann A, Neller S et al (2003) ISSLS prize winner: a novel approach to determine trunk muscle forces during flexion and extension: a comparison of data from an in vitro experiment and in vivo measurements. Spine 28:2585-2593

37. Woodard JC, Poulos PW Jr, Parker RB et al (1985) Canine diffuse idiopathic skeletal hyperostosis. Vet Pathol 22:317-326 